\title{
Herbs and Rehabilitation after Stroke Study: A Multi-center, Double-blinded, Randomized Trial in Hong Kong
}

\author{
Raymond Cheung, ${ }^{\mathrm{a}}$ Li Xiong, ${ }^{\mathrm{b}}$ Shek Kwan Chang, ${ }^{\mathrm{a}}$ Choi Ting Tse, ${ }^{\mathrm{a}}$ Yin Yu Pang, ${ }^{\mathrm{a}}$ Vincent Mok, \\ Thomas Leung, ${ }^{\mathrm{b}}$ Tak Hong Tsoi, ${ }^{\mathrm{c}}$ Richard Li, ${ }^{\mathrm{c}}$ May Mok, ${ }^{\mathrm{c}}$ Chee My Chang, ${ }^{\mathrm{d}}$ Kwok Kwong Lau, \\ Bun Sheng, ${ }^{\text {e }}$ Terrence Li, ${ }^{\mathrm{e}}$ Jonas Yeung, ${ }^{\mathrm{f}}$ Ping Chung Leung, ${ }^{\mathrm{g}}$ Ping Chook,, Ka Sing Wong ${ }^{\mathrm{b}}$ \\ aDepartment of Medicine, The University of Hong Kong, Hong Kong, Hong Kong \\ 'Department of Medicine and Therapeutics, The Chinese University of Hong Kong, Hong Kong, Hong Kong \\ 'Department of Medicine, Pamela Youde Nethersole Eastern Hospital, Hong Kong, Hong Kong \\ dDepartment of Medicine, Ruttonjee Hospital, Hong Kong, Hong Kong \\ 'Department of Medicine, Princess Margaret Hospital, Hong Kong, Hong Kong \\ fDepartment of Medicine, Alice Ho Miu Ling Nethersole Hospital, Hong Kong, Hong Kong \\ ${ }^{9}$ Institute of Chinese Medicine, The Chinese University of Hong Kong, Hong Kong, Hong Kong
}

\section{Dear Sir:}

Stroke is the leading cause of long-term adult disability throughout the world. ${ }^{1}$ Even with rehabilitation by physiotherapy and occupational therapy, about half of all stroke survivors are disabled with significant functional impairment. Thus, novel rehabilitation strategies are needed to supplement conventional therapies. An innovative herbal formula based on Traditional Chinese Medicine (TCM) literature for post-stroke rehabilitation using Huangqi (Radix Astragali, Inner Mongolia, 30g), Chuanxiong (Rhizoma Chuanxiong, Sichuan, 6g), Danshen (Radix Salviae Miltorrhizae, Sichuan, 20g), Juemingzi (Cassia Obtusifolia Linne, Jiangsu, 15g), Gancao (Radix Glycyrrhizae, Xinjiang, 6g), Danggui (Radix Angelicae Sinensiss, Gansu, 6g), Chishao (Paeoniae Veitchii Radix, Inner Mongolia, 15g) was demonstrated to be able to reduce the post-stroke brain damage by increasing the anti-oxidative status in vivo and in vitro rat model with transient middle cerebral artery occlusion. ${ }^{2}$ However, the protective effects of this formula on post-stroke recovery in humans remain unknown. We therefore conducted a randomized, double-blinded, placebo-controlled clinical trial to explore the potential efficacy and safety of this herbal formula in post-stroke rehabilitation.

This pilot study was conducted in 6 general hospitals with acute stroke admission in Hong Kong between 2006 and 2010, with ethics committee approval and written informed consent received at all participating centers. Inclusion criteria were: age $\geq 18$ years; diagnosis of ischemic stroke defined by the World Health Organization definition; previously independent in activities of daily living with a Barthel Index ${ }^{3}$ of 90 or more; a modified Rankin scale (mRS) ${ }^{4}$ of 0 or 1 before admission; a National Institutes of Health Stroke Scale (NIHSS) ${ }^{5}$ of 4 to 15 with a score of 2 or more in the limb motor items persisting beyond 48 hours but within 7 days of onset of stroke and availability for followups. In addition to conventional rehabilitation, patients were randomly assigned to one of the three treatment regimens: High dose group received active herbal preparation TCM for 8 weeks; Low dose group received active herbal preparation for 4 weeks, followed by placebo preparation for 4 weeks, and placebo group received placebo for 8 weeks. The primary outcome was mRS grade at month 3 , defined as a good outcome with a $m R S \leq 2$. Secondary outcomes were the scores of NIHSS, Barthel Index and Mini Mental State Examination (MMSE) at month 3 and 
Table 1. Primary outcome, secondary outcomes and mortality, adverse events and severe adverse events in each group

\begin{tabular}{|c|c|c|c|c|}
\hline & $\begin{array}{l}8 \text { weeks TCM } \\
(n=60)\end{array}$ & $\begin{array}{c}4 \text { weeks TCM }+4 \text { weeks } \\
\text { Placebo }(n=57)\end{array}$ & $\begin{array}{l}8 \text { weeks Placebo } \\
\quad(n=57)\end{array}$ & $P$ \\
\hline \multicolumn{5}{|l|}{ Primary outcome } \\
\hline $\mathrm{mRS} \leq 2$ at 3 months & $44(73.3 \%)$ & $36(63.2 \%)$ & $38(66.7 \%)$ & 0.434 \\
\hline \multicolumn{5}{|l|}{ Secondary outcome } \\
\hline $\mathrm{mRS} \leq 2$ at 6 months & $44(73.3 \%)$ & 40 (70.2\%) & 36 (63.2\%) & 0.439 \\
\hline NIHSS at 3 months & $3.0(1.8-4.3)$ & $3.0(1.0-5.0)$ & $3.0(1.0-5.0)$ & 0.846 \\
\hline NIHSS at 6 months & $2.0(1.0-4.0)$ & $3.0(2.0-5.0)$ & $3.0(1.8-5.0)$ & 0.331 \\
\hline $\mathrm{Bl}$ at 3 months & $95.0(85.0-100.0)$ & $95.0(65.0-100.0)$ & $95.0(75.0-100.0)$ & 0.413 \\
\hline $\mathrm{Bl}$ at 6 months & $100.0(85.0-100.0)$ & $95.0(75.0-100.0)$ & $100.0(85.0-100.0)$ & 0.713 \\
\hline MMSE at 3 months & $26.0(23.0-28.0)$ & $25.0(22.0-29.5)$ & $26.0(21.0-29.0)$ & 0.838 \\
\hline MMSE at 6 months & $27.0(24.0-29.0)$ & $26.0(21.5-29.0)$ & $25.5(21.3-30.0)$ & 0.333 \\
\hline \multicolumn{5}{|l|}{ Mortality } \\
\hline 3 months & $2(3.3 \%)$ & $0(0.0 \%)$ & $1(1.8 \%)$ & 0.502 \\
\hline 6 months & $4(6.7 \%)$ & $0(0.0 \%)$ & $2(3.5 \%)$ & 0.366 \\
\hline \multicolumn{5}{|l|}{ Vascular events at 6 months } \\
\hline Recurrent stroke & $2(3.3 \%)$ & $2(3.5 \%)$ & $4(7.0 \%)$ & 0.347 \\
\hline Acute coronary syndrome & $1(1.7 \%)$ & $2(3.5 \%)$ & $0(0.0 \%)$ & 0.502 \\
\hline Deep vein thrombosis & $0(0.0 \%)$ & $1(1.8 \%)$ & $0(0.0 \%)$ & 0.983 \\
\hline $\begin{array}{l}\text { Haemorrhagic transformation or } \\
\text { intracerebral hemorrhage }\end{array}$ & $1(1.7 \%)$ & $1(1.8 \%)$ & $0(0.0 \%)$ & 0.404 \\
\hline Adverse event & $26(43.3 \%)$ & $31(54.4 \%)$ & $25(43.9 \%)$ & 0.939 \\
\hline Severe adverse events & $14(23.3 \%)$ & 11 (19.3\%) & $9(15.8 \%)$ & 0.305 \\
\hline
\end{tabular}

mRS, modified Rankin scale; NIHSS, National Institutes of Health Stroke Scale; BI, Barthel Index; MMSE, mini Mental State Examination; TCM, Traditional Chinese Medicine.

month 6, and mRS at month 6 .

Group differences for continuous data and non-normally distributed data were examined by ANOVA test or Kruskal-Wallis test, respectively. Intention-to-treat analysis was performed in all randomized patients. All statistical analysis was performed with SPSS version 16.0 (SPSS Inc., Chicago, IL, USA). Differences with $P<0.05$ were considered significant.

Of the randomized 198 patients, totally 24 patients were lost to follow-up at month 3. Therefore, 174 patients (87.9\%) were included the intention-to-treat analysis (60 patients with high dose TCM, and 57 with low dose TCM and placebo, respectively). The groups were balanced in most demographic data except the prevalence of ischemic heart disease $(P=0.024)$. The proportion of patients with $\mathrm{mRS} \leq 2$ at month 3 was $73.3 \%$ in the TCM group and 66.7\% in the placebo group as shown in Table 1 (absolute risk reduction $6.6 \%, P=0.434)$. There were no significant differences in the secondary outcomes, the mortality rate, the occurrence of vascular events by 6 months (recurrent stroke, acute coronary syndrome, deep vein thrombosis and hemorrhagic transformation or intracerebral hemorrhage), adverse events, or severe adverse events across the groups (Table 1 , all $P>0.05$ ).

Our study showed a favorable trend that $73.3 \%$ of patients in the herbal formula group had good outcomes at 3 months after stroke onset, which was slightly higher than those in the placebo group. The favorable trend might warrant further investigation into the use of this herbal formula for ischemic stroke rehabilitation. Additionally, the results showed a very good safety profile for the use of herbal formula. Overall the treatment was very well tolerated and none of the adverse events were considered drug-related.

Currently, there are more than 100 TCM agents used clinically in China for stroke with the approval of the Chinese National Drug Administration. ${ }^{6}$ However, there is a lack of availability of the evidence for the efficacy and safety of TCM. A recent metaanalysis ${ }^{6}$ of TCM for ischemic stroke found only 3 studies were randomized, double-blind, and placebo-controlled, and only 2 studies had long-term outcome assessment. In Asia, a doubleblind, placebo-controlled, randomized, multi-center study to investigate Chinese medicine Neuroaid efficacy on stroke recovery (CHIMES Study) is ongoing to test whether Neuroaid is superior to placebo in reducing neurological deficit and improving functional outcome in patients with acute cerebral infarction of an intermediate severity. The sub-study of CHIMES confirmed the safety of Neuroaid in acute stroke patients receiving a 3-month treatment. ${ }^{8}$ Different from TCM used in previous studies, we developed an innovative herbal formula with 7 components, in which Huangqi and Danshen are the main ingredients. Regarding the underlying mechanisms of TCM in post-stroke recovery, it may be attributed to antioxidant, anti-inflammatory, and antiglutamate effects of TCM, which could dilate blood vessels, sup- 
press platelet aggregation, protect against ischemic reperfusion injury, and enhance the tolerance of ischemic tissue to hypoxia. ${ }^{10}$

Our study had the limitation of having a small sample size, which may lead the non-significant difference on the efficacy of the herbal formula in post-stroke recovery treatment. Nevertheless, our study's strength is that some favorable trends were observed and results provided estimates for sample size requirements to achieve statistical significance in future large-scale clinical studies.

In conclusion, the innovative herbal formula for 8-week medication did not statistically significantly facilitate neurological recovery in ischemic stroke beyond 48 hours but within 7 days of onset. However, some favorable trend could be noted on the $\mathrm{mRS}$ score in the herbal formal group versus the placebo, which probably need a large phase III trial to confirm the preliminary findings.

\section{References}

1. Liu L, Wang D, Wong KS, Wang Y. Stroke and stroke care in china: huge burden, significant workload, and a national priority. Stroke 2011:42:3651-3654.

2. Koon CM, Chan CK, Lee KY, Chan YL, Lam MY, Wong YL, et al. Anti-oxidative properties of post-stroke recovery formula in preventing transient MCAo-induced brain damage. Prog Nutr 2010;12:74[abstract].

3. Mahoney Fl, Barthel DW. Functional evaluation: the Barthel index. Md State Med J 1965;14:61-65.

4. de Haan R, Limburg M, Bossuyt $P$, van der Meulen J, Aaronson $N$. The clinical meaning of rankin 'handicap' grades after stroke. Stroke 1995;26:2027-2030.

5. Brott T, Adams HP Jr, Olinger CP, Marler JR, Barsan WG, Biller $J$, et al. Measurements of acute cerebral infarction: a clinical examination scale. Stroke 1989;20:864-870.

6. Wu B, Liu M, Liu H, Li W, Tan S, Zhang S, et al. Meta-analysis of traditional Chinese patent medicine for ischemic stroke. Stroke 2007;38:1973-1979.

7. Venketasubramanian $N$, Chen $C L$, Gan RN, Chan BP, Chang HM, Tan SB, et al. A double-blind, placebo-controlled, randomized, multicenter study to investigate CHInese Medicine Neuroaid Efficacy on Stroke recovery (CHIMES Study). Int J Stroke 2009;4:54-60.

8. Young $S H$, Zhao Y, Koh A, Singh R, Chan BP, Chang HM, et al. Safety profile of MLC601 (Neuroaid) in acute ischemic stroke patients: a Singaporean substudy of the Chinese medicine neuroaid efficacy on stroke recovery study. Cerebrovasc Dis 2010;30:1-6.

9. Rausch WD, Liu S, Gille G, Radad K. Neuroprotective effects of ginsenosides. Acta Neurobiol Exp (Wars) 2006;66:369-375.

10. Bei W, Peng W, Zang L, Xie Z, Hu D, Xu A. Neuroprotective effects of a standardized extract of diospyros kaki leaves on MCAO transient focal cerebral ischemic rats and cultured neurons injured by glutamate or hypoxia. Planta Med 2007;73: 636-643.

Correspondence: Ka Sing Wong

Department of Medicine \& Therapeutics, the Chinese University of Hong Kong, 30-32 Ngan Shing street, Shatin, Hong Kong, 999077.

Tel: +852-2632-3144

Fax: +852-2649-3761

Email: ks-wong@cuhk.edu.hk

Received: July 26, 2016

Revised: July 26, 2016

Accepted: July 28, 2016

This study was funded by an Innovation and Technology Support Programme (reference no. GHP/037/05 - Modernization of Chinese Medicine: From Clinical Efficacy to Drug Production - Two Innovative Formulae for comprehensive Research).

The authors have no financial conflicts of interest. 